\title{
Comparative Study on Classification of Spun Silk Fabrics for Shirt
}

\author{
Caiyuan KUANG \\ College of Art, Jinling Institute of Technology, Nanjing, Jiangsu, China (kcy@jit.edu.cn)
}

\begin{abstract}
In this paper, mechanical properties of tensile, shear and bending of 28 different kinds of spun silk fabrics for shirt were tested by using KES. Classification of those fabrics was carried out by using methods of three-group discriminance and system cluster analysis. And the differences on mechanical properties and specification parameters of classified fabrics were discussed. The results showed that the methods of three-group discriminance and system cluster analysis can be effective to classify those spun silk fabrics. However, system cluster analysis is superior to three-group discriminance, owning convenient operation and efficiency property. The results also indicated that those fabrics were classified as leisure shirt fabrics and full-dress shirt fabrics. And there were significant differences in main mechanical properties and specification parameters between the two classified fabrics. It will provide quantitative references for the design of spun silk fabrics.
\end{abstract}

Keywords $\rightarrow$ spun silk fabric, mechanical properties, three-group discriminance, system cluster analysis

\section{绢纺衬衫面料分类的比较研究}

\author{
匡才远 \\ 金陵科技学院艺术学院, 南京, 江苏, 中国
}

摘 要 本文利用 KES 测量了 28 种绢纺祄衫面料的主要力学性能, 运用三群判别公式法和系统聚类分析法进行分类研究, 探讨 了面料的力学性能与规格参数的差异。结果表明, 三群判别公式法与系统聚类分析法对绢纺祄衫面料的分类具有较高的相似性, 系统 聚类分析则方便易操作, 分类效果好。所选面料可分为休闲祄衫面料和正装祄衫面料两类, 两类面料的主要力学性能指标及规格参数 具有明显的差异，可为企业设计不同用途的面料提供量化依据。

关键词 绢纺面料, 力学性能, 三群判别公式, 系统聚类分析

1. 引言

随着流行趋势的发展, 衬衫应用的范围变得更加广泛, 既可以作为生活便装, 也可作为礼服出现在正式场合。目 前, 人们只是定性地判断面料适合于某种类型的服装, 没 有定量地分析面料与服装款式类别之间的关系。随着计算 机技术的发展, 计算机视觉技术引入到织物自动分类识别 中, 提高了织物识别效率, 降低了成本。常用的计算机视 觉织物分类技术大致可以分为: 基于统计模式识别方法, 基于模糊模式识别方法, 基于最近邻分类方法, 基于神经 网络方法, 基于贝叶斯方法, 基于小波变换方法等技术 [1]。 此外, 研究人员利用聚类方法对绸型裙装面料 [2]、弹性面 料[3]进行了分类研究。
本文以 28 种绢纺祄衫面料为研究对象, 利用 KES 织物 风格仪测量了面料的拉伸、剪切、弯曲等力学性能, 运用 三群判别公式法和系统聚类分析法对面料进行分类研究, 并探讨了不同类型绢纺祄衫面料的力学性能与规格参数的 异同性。

\section{2. 实验材料与方法}

\section{1 实验材料}

选取 28 种绢纺面料为实验材料, 面料均为绢与棉、麻、 毛、粘胶、竹等纤维混纺的素色、条格机织物。 


\section{2 实验方法}

将绢纺面料裁剪成 $20 \mathrm{~cm} \times 20 \mathrm{~cm}$ 的试样, 保持试样的平 整性, 在恒温恒湿实验室（温度 $\mathrm{T}=20 \pm 2^{\circ} \mathrm{C}$, 相对湿度 $\mathrm{RH}$ $=65 \pm 5 \%$ ）中平衡 24 小时。

运用 KES 织物风格仪测试绢纺面料的拉伸性能、剪切 性能、弯曲性能等力学指标, 包括拉伸线性度 $\mathrm{L}_{\mathrm{T}}$ 、拉伸最 大应变率 EMT、弯曲刚度 B、弯曲滞后矩 $2 \mathrm{HB}$ 、剪切刚度 $\mathrm{G}$ 、 $5^{\circ}$ 剪切滞后矩 $2 \mathrm{HG}_{5}$ 。运用 B52245 电子天平测量单位面积 重量 W。

\section{3. 实验结果分析}

\section{1 三群判别公式法分类}

三群判别公式是日本绫田等学者提出的, 其原理是根 据面料的拉伸线性度、最大拉伸应变率、剪切刚度、剪切 滞后矩、弯曲刚度、弯曲滞后矩、单位面积重量等指标, 运用判别公式, 参照三群判别公式参数表, 分别计算出判 别值 $Z_{1} 、 Z_{2}$, 进而对各种面料进行分类 [4]。三群判别公式 如下:

$$
\begin{aligned}
& Z_{1}=\sum_{i=1}^{7} C_{1 i} X_{i} ; \quad Z_{2}=\sum_{i=1}^{7} C_{2 i} X_{i} ; \\
& X_{i}=\left(x_{i}-m_{i}\right) / \sigma_{i} \quad(\text { 公式 I ) }
\end{aligned}
$$

其中, $Z_{1} 、 Z_{2}$ 指判别值; $C_{1 i} 、 C_{2 i}$ 为常数; $X_{i}$ 指标准化后 的力学指标; $\mathrm{x}_{\mathrm{i}}$ 指基本力学指标; $\mathrm{m}_{\mathrm{i}}$ 指 $\mathrm{x}_{\mathrm{i}}$ 的平均值; $\sigma{ }_{\mathrm{i}}$ 指 $\mathrm{x}_{\mathrm{i}}$ 的标准差。

利用公式 I , 绫田等学者根据对制服类、悬垂类和宽 松挺括类等 3 类 125 种服装面料的测定结果，得到不同服 装面料的类别判别图, 如图 1 所示。

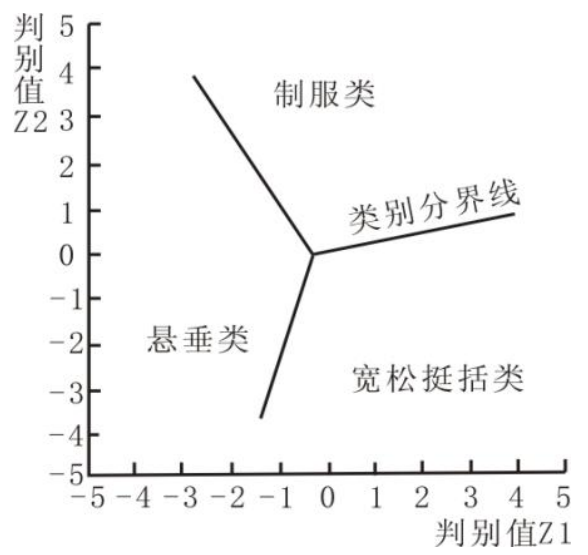

图 1 服装面料类别判别图
运用公式 I 计算出本文所测的 28 种绢纺衬衫面料的 判别值 $Z_{1} 、 Z_{2}$, 并以此判别值为横纵坐标, 28 种绢纺面料 的分布如图 2 所示。

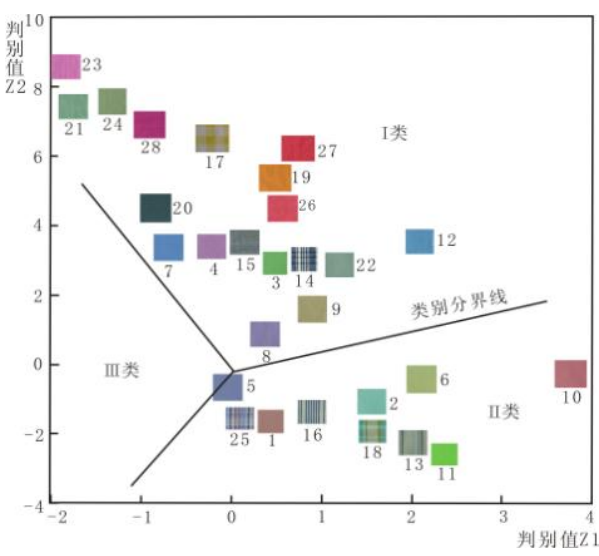

图 2 绢纺面料的分类判别图

28 种绢纺面料划分成两大类, 即 I 类 $=\{$ 包含 23,21 , $24,28,17,20,7,27,19,26,4,15,3,14,22,12$, $9,8$ 号试样 $\}$, II 类 $=\{$ 包含 $5,6,10,25,1 ， 16 ， 2 ， 18$, 13, 11 号试样\}。

\section{2 系统聚类法分类}

用 $d_{i j}$ 表示样品 $i$ 和样品 $j$ 的距离, $G_{1}, G_{2}, \cdots$ 表示类, 用 $D_{p q}$ 表示 $G_{P}$ 与 $G_{q}$ 的距离, 则

$$
D_{p q}=\min _{i \in G_{p}, j \in G_{q}} d_{i j}
$$

用最小距离法聚类的步骤如下:

(1) 规定样品之间的距离, 计算样品两两距离的对称 阵, 记作 $D_{(0)}$ 。显然 $D_{p q}=d_{p q}$ 。

(2) 选择 $D_{(0)}$ 的最小元素, 设为 $D_{p q}$, 则将 $G_{P}$ 和 $G_{q}$ 合 并成一新类, 记为 $G_{r}, G_{r}=\left\{G_{p}, G_{q}\right\}$ 。

（3）计算新类与其他类的距离

$$
\begin{aligned}
G_{r k}=\min _{i \in G_{r}, j \in G_{k}} d_{i j} & =\min \left\{\min _{i \in G_{p}, j \in G_{k}} d_{i j}, \min _{i \in G_{q}, j \in G_{k}} d_{i j}\right\} \\
& =\min \left\{D_{p k}, D_{q k}\right\}
\end{aligned}
$$


将 $D_{(0)}$ 中的 $p, q$ 行, $p, q$ 列用 (2) 并成一个新行新列, 新行新列对应 $G_{r}$, 所得到的矩阵记作 $D_{(1)}$ 。

(4) 对 $D_{(0)}$ 重复上述对 $D_{(0)}$ 的两步得 $D_{(2)}$, 如此下去直 到所有的元素成为一类为止。

28 种面料的分析结果如图 3 所示。

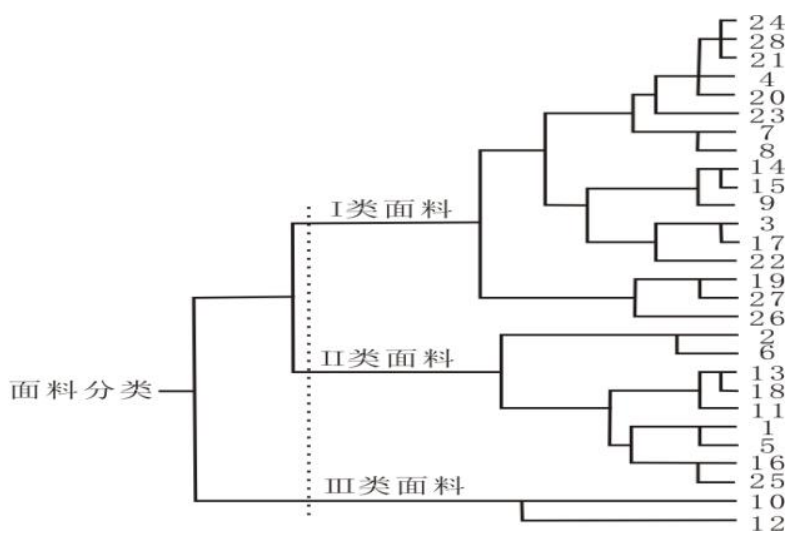

图 3 绢纺面料聚类分类图

从聚类分类图中可知, 如果将 28 种实验面料分成 3 个
聚类域, 则 I 类由 $24,28,21,4,20,23,7,8,14$, $15,9,3,17,22,19,27,26$ 号试样面料组成, II 类由 $2,6,13,18,11,1,5,16,25$ 号试样面料组成, III类 由 10,12 号试样面料组成。

对比上述两种方法分类结果, 发现两种方法的分类结 果相似, 只是按照系统聚类分析, 面料 10 号和 12 号归为 单独的一类, 其余面料的分类结果与运用三群判别公式法 得到的结果一致。由图 2 可以看出, 面料 10 号和 12 号的 位置相对而言处于边缘位置, 10 号和 12 号面料的纬纱由 $41.67 \mathrm{tex} \times 1$ 的亚麻纱线构成, 面料外观粗犷挺括, 具有 独特的外观风格。

图 2、图 3 的分析结果表明, 上述两种分类方法都可 以有效地进行绢纺祄衫面料的分类, 但三群判别公式法进 行面料分类需要参照参数表计算判别值, 计算工作量比较 大, 而借助统计软件 SPSS 进行绢纺衬衫面料的系统聚类分 析则方便易操作, 分类速度快。

\section{3 两类面料力学性能差异分析}

I 、II 两类面料力学指标的差异性可以通过统计学中 的 $\mathrm{F}$ 检验来区分, $\mathrm{F}$ 检验是用来区分两个总体之间差异性 的方法, F 值越大, 表示两个总体之间的差异越显著。I、 II 两类面料力学指标的平均值及 $\mathrm{F}$ 检验的结果如表 1 所示。

表 1 两类绢纺面料力学指标

\begin{tabular}{|l|c|c|c|c|c|c|c|}
\hline & 拉伸线性度度 $\mathrm{L}_{\mathrm{T}}$ & 最大拉伸应变率 $\mathrm{EMT}$ & 弯曲刚度 $\mathrm{B}$ & 弯曲滞后矩 $2 \mathrm{HB}$ & 剪切刚度 $\mathrm{G}$ & 剪切滞后矩 $2 \mathrm{HG}_{5}$ & 平方米克重 $\mathrm{W}$ \\
\hline $\begin{array}{l}\text { I 类平 } \\
\text { 均值 }\end{array}$ & 0.593 & 5.846 & 0.152 & $8.70 \mathrm{E}-02$ & 0.268 & 0.327 & 15.03 \\
\hline $\begin{array}{l}\text { II 类平 } \\
\text { 均值 }\end{array}$ & 0.619 & 4.197 & 0.100 & $7.50 \mathrm{E}-02$ & 0.605 & 2.092 & 14.48 \\
\hline F 值 & 1.329 & 3.312 & 5.847 & 0.691 & 136.609 & 210.845 & 0.179 \\
\hline Sig. & 0.260 & 0.081 & 0.024 & 0.414 & 0.000 & 0.000 & 0.676 \\
\hline
\end{tabular}

表1分析结果表明, I 类、II 类绢纺面料的最大拉伸应 变率、弯曲刚度、剪切刚度及剪切滞后矩等力学指标差异 的显著性水平小于 0.1 , 因此, 两类面料相应的力学性能指 标存在显著性差异, 这是绢纺祄衫面料按照力学性能分为 两类的依据。

根据力学指标的特征含义可知, B表示织物的刚柔性, $\mathrm{B}$ 值越小, 织物的柔性越好, $\mathrm{B}$ 值越大, 织物的刚性越好。 表1结果还表明 I 类面料的刚性优于 II 类面料的刚性, 说明 I 类面料满足休闲男祄衫的风格要求, II 类面料满足正装
男祄衫的风格要求。

\section{4 两类面料规格参数的分析}

为进一步分析两类面料产生差异的原因, 将 I、II 类 面料的经纱线密度、纬纱线密度、织物经向密度、织物纬 向密度、混纺比等规格参数进行分析, 从而为绢纺面料的 设计提供参考依据。I 、II 两类面料的规格参数如表 2,3 所示。 
表 2 I 类绢纺面料的规格参数

\begin{tabular}{|c|c|c|c|c|c|}
\hline 编号 & 经纱线密度 & 纬纱线密度 & 经密 & 纬密 & 混纺比 \\
\hline 3 & 8. 33 tex $\times 2$ & 27. 78 tex $\times 1$ & 37 & 26.5 & 51丝/49麻 \\
\hline 4 & 16. 67 tex $\times 2$ & $\begin{array}{c}12.5 \text { tex } \times 2 \\
35.71 \text { tex } \times 1\end{array}$ & 31 & 26.5 & 75 丝/25麻 \\
\hline 7 & 8. 33 tex $\times 2$ & 8. 33 tex $\times 2$ & 54 & 31 & 75 丝/25毛 \\
\hline 8 & 8. 33 tex $\times 2$ & $\begin{array}{l}\text { 8. } 33 \text { tex } \times 2 \\
27.78 \text { tex } \times 1\end{array}$ & 34 & 24 & 70 丝/30麻 \\
\hline 9 & 7. 14 tex $\times 2$ & 20. 00 tex $\times 1$ & 40 & 33.5 & 48丝/52麻 \\
\hline 14 & 24. 39 tex $\times 1$ & 27. 03 tex $\times 1$ & 30 & 25 & 56丝/44麻 \\
\hline 15 & 22. 22 tex $\times 1$ & 24. 39 tex $\times 1$ & 30 & 25 & 55 丝/45麻 \\
\hline 17 & 22. 22 tex $\times 1$ & 22. $22 \operatorname{tex} \times 1$ & 30 & 26 & 56 丝/44麻 \\
\hline 19 & 16. 67 tex $\times 2$ & 33. 33 tex $\times 1$ & 36 & 32 & 52 丝/48麻 \\
\hline 20 & 19. 23 tex $\times 2$ & 33. 33 tex $\times 1$ & 27 & 35 & 57 丝/43麻 \\
\hline 21 & 19. 23 tex $\times 2$ & 19. 23 tex $\times 2$ & 22 & 22 & 42丝/58麻 \\
\hline 22 & 8.33 tex $\times 2$ & 24. 39 tex $\times 1$ & 38 & 32 & 51 丝/49麻 \\
\hline 23 & 8. 33 tex $\times 2$ & 19. 23 tex $\times 1$ & 54 & 30 & 55丝/45麻 \\
\hline 24 & 33. 33 tex $\times 1$ & 16. 67 tex $\times 2 \quad 33.33$ tex $\times 1$ & 24 & 24 & 40丝/60麻 \\
\hline 26 & 10. 00 tex $\times 2$ & 22. 22 tex $\times 1$ & 45 & 34 & 52丝/48麻 \\
\hline 27 & 13. 52 tex $\times 2$ & 33. 33 tex $\times 1$ & 30 & 30 & 38丝/62麻 \\
\hline \multirow[t]{2}{*}{28} & 9. 62 tex $\times 2$ & 24. 39 tex $\times 1$ & 45 & 28 & 38丝/62麻 \\
\hline & 24. 09 & 26. 62 & 35.7 & 28.5 & \\
\hline
\end{tabular}

表 2,3 分析结果表明, I 类面料经纬纱线的线密度平 均值略大于 II 类面料, 而 II 类面料的经纬密度大于 I 类面 料, II 类面料中绢丝的比例高于 I 类面料。可见, I 类面
料具有纱线较粗、结构相对疏松、面料外观粗犷挺括的特 征, 体现了休闲祄衫风格; 而 II 类面料的纱线较细、外观 风格细淢、光泽优雅, 满足正装衬衫风格的要求。

\section{表 3 II 类绢纺面料的规格参数}

\begin{tabular}{|c|c|c|c|c|c|}
\hline 编号 & 经纱线密度 & 纬纱线密度 & 经密 & 纬密 & 混纺比 \\
\hline 1 & $10.00 \mathrm{tex} \times 2$ & $10.00 \mathrm{tex} \times 2$ & 40.5 & 29 & 70 丝 $/ 30$ 棉 \\
\hline 2 & $16.67 \mathrm{tex} \times 2$ & $31.25 \mathrm{tex} \times 1$ & 21.5 & 44.5 & 35 丝 $/ 65$ 棉 \\
\hline 5 & $27.78 \mathrm{tex} \times 1$ & $37.04 \mathrm{tex} \times 1$ & 28.5 & 20.5 & 50 天丝 $/ 50$ 粘胶 \\
\hline 6 & $12.5 \mathrm{tex} \times 2$ & $18.52 \mathrm{tex} \times 1$ & 43 & 31 & 72 丝 $/ 28$ 棉 \\
\hline 11 & $8.33 \mathrm{tex} \times 2$ & $8.33 \mathrm{tex} \times 2$ & 38 & 29 & 75 绢 $/ 25$ 竹 \\
\hline 13 & $7.58 \mathrm{tex} \times 2$ & $6.58 \mathrm{tex} \times 2 \quad 24.39 \mathrm{tex} \times 1$ & 35 & 30 & 60 丝 $/ 20$ 麻 $/ 20$ 棉 \\
\hline 16 & $22.22 \mathrm{tex} \times 1$ & $15.15 \mathrm{tex} \times 1$ & 30 & 26 & 72 丝 $/ 28$ 麻 \\
\hline 18 & $8.33 \mathrm{tex} \times 2$ & $8.33 \mathrm{tex} \times 224.39 \mathrm{tex} \times 1$ & 36 & 36 & 55 丝 $/ 45$ 棉 \\
\hline 25 & $19.23 \mathrm{tex} \times 1$ & $15.15 \mathrm{tex} \times 1$ & 36.1 & 30.2 & \\
\hline & 21.78 & 21.45 & & \\
\hline
\end{tabular}




\section{4. 结论}

(1) 三群判别公式法与系统聚类分析法对绢纺祄衫面 料的分类具有较高的相似性。但三群判别公式法进行织物 分类需要参照参数表计算判别值, 计算量比较大, 而借助 统计软件 SPSS 进行系统聚类分析则方便易操作, 分类速度 快, 效果较好。

(2) 28 种绢纺面料大致可以分为休闲祄衫面料及正 装祄衫面料。休闲衬衫面料的外观纹路比较粗犷, 表面有 突起或颗粒; 正装男祄衫面料的外观纹路细淢, 光泽柔和, 表面光滑, 毛羽少。

(3) 两类面料在最大拉伸应变率、弯曲刚度、剪切刚 度及剪切滞后矩等力学性能指标方面存在显著性差异。I 类面料的纱线较粗且组织结构略松散, 丝含量较低; II 类 面料的纱线较细且组织结构较致密, 丝含量较高。两类面 料在力学性能与规格参数方面的差异可以为绢纺面料的设
计提供参考依据。

\section{参考文献(References)}

[1] Z. M. Wu, T. Lv and J. W. Wang, "Study on intelligent evaluation of fabric style using fuzzy expert systems," Journal of Textile Research, vol.26, no.4, pp.115-117, 2005.

[2] Q. P. Zhang, C. Y. Kuang and F. Qin, "Study on classification of spun silk fabric for skirt," Journal of Tianjin Polytechnic University, vol.29, no.4, pp.25-28, 2009.

[3] P. Zheng and W. Tuo, "Study on classification of elastic fabric for pant based on K-means clustering algorithm," Melliand China, no.5, pp.71-76, 2014.

[4] F. M. Wang. Performance Design of Garment Material. Shanghai: China Textile University Press, 2000. 